\title{
Optimization of Fluctuation Suppression in Photovoltaic Power Generation Using Gas Cogeneration System
}

\author{
Manimel Wadu Saminda Roshan, Yasunori Mitani, Masayuki Watanabe, and Yaser Qudiah
}

\begin{abstract}
This research aims to design a frequency power system control model in order to suppress frequency fluctuations in the power system in case of mass introduction of photovoltaic generation to the power system. The effectiveness of the proposed control model is demonstrated though simulation in MATLAB/SIMULINK environment. In this research, photovoltaic power fluctuation suppression by combined heat and power system (generally known as Cogeneration system) is analyzed in detail based on a simulation model corresponding to a real scale industry power system, which consists of a domestic photovoltaic power system and gas cogeneration system.
\end{abstract}

Index Terms-Photovoltaic power, cogeneration system, optimization, power fluctuation, LFC.

\section{INTRODUCTION}

Energy is expected to play a vital role in supporting standards of living. Our living standards could not be fulfilled without energy. Economic development and technical progress are often connected with the quantity as well as quality of energy used. As the consumption of energy goes up, energy crises and environmental problems continues to be a major topic in energy engineering [1], [2]. In Japan, due to lack of significant domestic energy sources such as fossil fuel, coal, crude oil has led to an increased need for energy production from renewable energy sources and due to global warming and environmental protection, Japan as well as the whole world is going into renewable energy sources such as photovoltaic power generation, wind power generation. This will be introduced into a power system in large quantities in different areas. It is expected in Japan that photovoltaic power is among the rapidly spreading renewable energy sources in the past few years [3]. It is being considered as a front-runner in process of clean energy. Major factors of increasing utilization of photovoltaic include increasing demand of energy, increasing environmental concern and energy security. However in case of sudden change in energy demand as well as energy supply, the power system might experience frequency fluctuations which need to be damped out. If these fluctuations are not damped out, there might have a bad influence on the frequency which can affect the power quality. The power of the natural energy sources such as photovoltaic power and wind power fluctuates depending on the weather conditions. Moreover, increased penetration of those unstable energy sources especially in isolated system may cause

Manuscript received July 2, 2015; revised September 29, 2015.

The authors are with the Department of Electrical and Electronic Engineering, Kyushu Institute of Technology, Japan (e-mail: p349538w@mail.kyutech.jp). unusual frequency derivation. Because of introducing more renewable generation for the national grid, balancing grid frequency is becoming a huge challenge for the grid. If it particularly sunny day and generate electricity from PV can change frequency in at high rate. If it is opposite, it can be also equally problematic which can cause frequency to fall at a faster rate. On the other hand, the introduction of the controllable distributed power systems such as the gas cogeneration systems are also increasing in recent years. Cogeneration (cogen) comes from Combined Heat and Power, which is a simultaneous process of producing heat and electricity [4], [5]. Different kind of fuels can be used to facilitate cogeneration. In this research, gas is used as the fuel to facilitate cogeneration. Cogeneration is high efficiency system which can save energy approximately $40 \%$ compared to produce electricity in separately. As an on-site power production system, the high efficiency cogeneration provides a number of benefits including reduced transportation and distribution loss which help to reduce energy costs. Thus, the main objective of this paper is to evaluate the effects of the controllable distributed systems which contributes to the suppression of power fluctuation in domestic photovoltaic power systems. MATLAB/SIMULINK environment is utilized for the simulation and results. In this paper, Load Frequency Control (LFC) problem is considered mainly when applying PVs in to power system on a large scale. Due to lack of capacitance of the power system, load frequency power system model is introduced to overcome LFC problem (see Fig. 1).

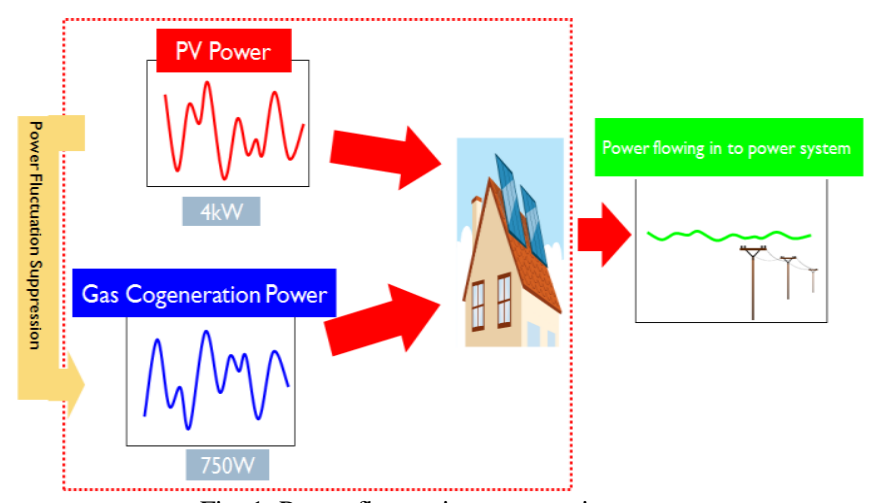

Fig. 1. Power fluctuation suppression system.

\section{MODELING OF SYSTEM DYNAMICS}

In this section, a simple frequency power system model for a household power system which contains PV and Gas cogeneration system is described [6].

The linear model of the power system, including governor and turbine is shown in Fig. 2 and Table $\mathrm{I}$, where $\Delta f$ is the 
frequency deviation of power system is defined by

$$
\Delta f=\frac{\Delta P_{e}-\Delta P_{L}}{D}
$$

TABLE I: DYNAMIC CONSTANTS OF POWER SYSTEM

\begin{tabular}{|l|l|}
\hline Inertia constant [p.u.MW.s/Hz] & $M=0.2$ \\
\hline Control factor [p.u.MW/Hz] & $D=0.008$ \\
\hline Turbine ratio & $K_{r}=0.333$ \\
\hline Turbine time constant [s] & $T_{r}=10$ \\
\hline Turbine time constant [s] & $T_{T}=0.3$ \\
\hline Governor time constant [s] & $T_{g}=0.2$ \\
\hline Permanent regulation [Hz/p.u.MW] & $R=2.5$ \\
\hline Bias coefficient [p.u.MW/rad] & $B=0.2$ \\
\hline Integral coefficient [1/s] & $K_{i}=0.03$ \\
\hline Bias coefficient [p.u.MW/Hz] & $K_{T}=0.6 / 3.3$ \\
\hline
\end{tabular}

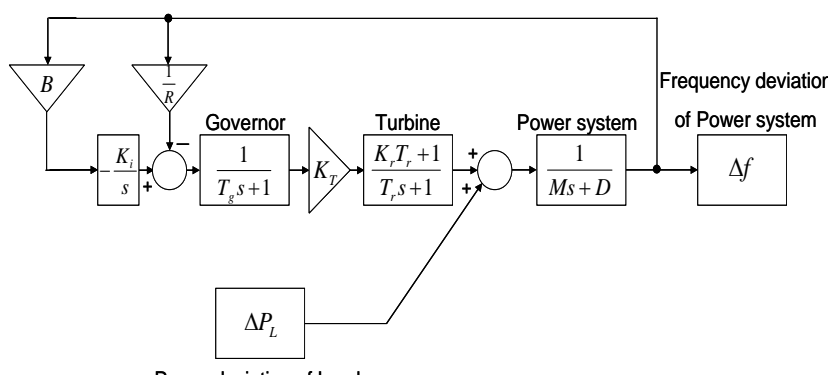

Power deviation of Load

Fig. 2. The block diagram of a linear power system.

This diagram shows the frequency derivation of the power system during load changes. The consequences of the above will be a corresponding perturbation in the frequency supply. When PV applies the combination of the linear power system and gas cogeneration system is shown in Fig. 3 and Table II where:

TABLE II: CONSTANTS OF GAS COGENERATION MODEL

\begin{tabular}{|l|l|}
\hline Inertia constant [p.u.MW.s/Hz] & $M_{O}=0.08$ \\
\hline Damping factor [p.u.MW/Hz] & $D_{O}=0.008$ \\
\hline Governors time constant [s] & $T_{g o}=0.2$ \\
\hline Permeant speed regulation [Hz/p.u.] & $R_{0}=3.02$ \\
\hline Synchronizing coefficient [p.u.MW.s/rad] & $T=0.35$ \\
\hline Capacitance ratio of power system & $a=0.0000027$ \\
\hline
\end{tabular}

As to overcome LFC problems that described in the diagram, here it is added a High-pass filter to filter the fluctuation components which only belongs to LFC frequency range, where:

$$
H(s)=\frac{s}{s+\omega}
$$

$\omega$ is the angular velocity is defined by

$$
\omega=2 \pi f=\frac{2 \pi}{T}[\mathrm{rad} / \mathrm{sec}]
$$

High-pass filter extracts the fluctuation components of the $\mathrm{PV}$, which only belongs to LFC frequency range [7]. Once the components are extracted, it goes to gas cogeneration system where fluctuation suppression performs. In the gas cogeneration system, it makes a negative wave compared to the wave, which come through high-pass filter. This makes to cancel the fluctuation components of each other and makes a new wave, which is more stable and smooth. Generally, gas cogeneration runs under the rated output. Combine it with PV and use it for the suppression of PV at full power makes a vast efficiency loss in gas cogeneration. So, here it is assumed that gas cogeneration control is performed in a range of $\pm 0.15 \mathrm{~kW}$ from $0.6 \mathrm{~kW}$. Another main part that has to be considered is when PV is too bigger compared to gas cogeneration, it is difficult to suppress all the fluctuation components of PV. Therefore, shrinkage factor is added in-between high-pass filter and gas cogeneration before filtered wave goes in to gas cogeneration.

The fluctuation components of PV will be shrunk at the shrinkage factor, which does not exceed the capacitance limit $(0.3 \mathrm{~kW})$ of gas cogeneration. Therefore, the gas cogeneration can suppress all the fluctuation components of PV.

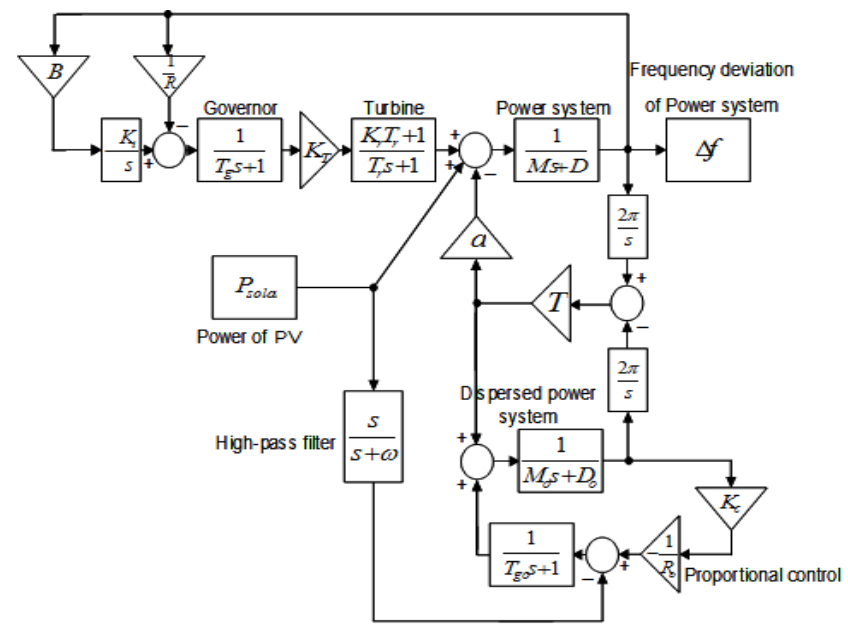

Fig. 3. Combination model of linear power system and gas cogeneration when PV applies (Load frequency power system model).

\section{SimUlation RESUltS AND DisCUSSION}

Computer simulations have been carried out in order to validate the effectiveness of the proposed model. The MALLAB/SIMULINK software package has been used for this purpose. The results of Fig. 4 and Fig. 5 show the difference of suppress process of gas cogeneration system with and without a shrinkage factor to shrink the fluctuation components of PV.
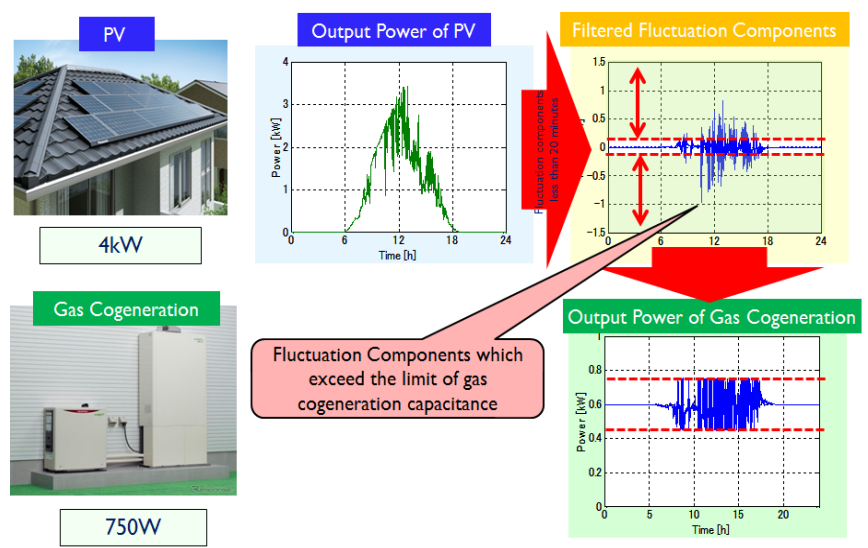

Fig. 4. The gas cogeneration system without a shrinkage factor in load frequency power system model. 
The gas cogeneration system without a shrinkage factor in the model PV fluctuation components are too big to suppress in gas cogeneration. It can only suppress some of the fluctuation components, not all. But adding a shrinkage factor to the model as shown in Fig. 5, gas cogeneration can suppress all the components of the PV that come through the high-pass filter [8].
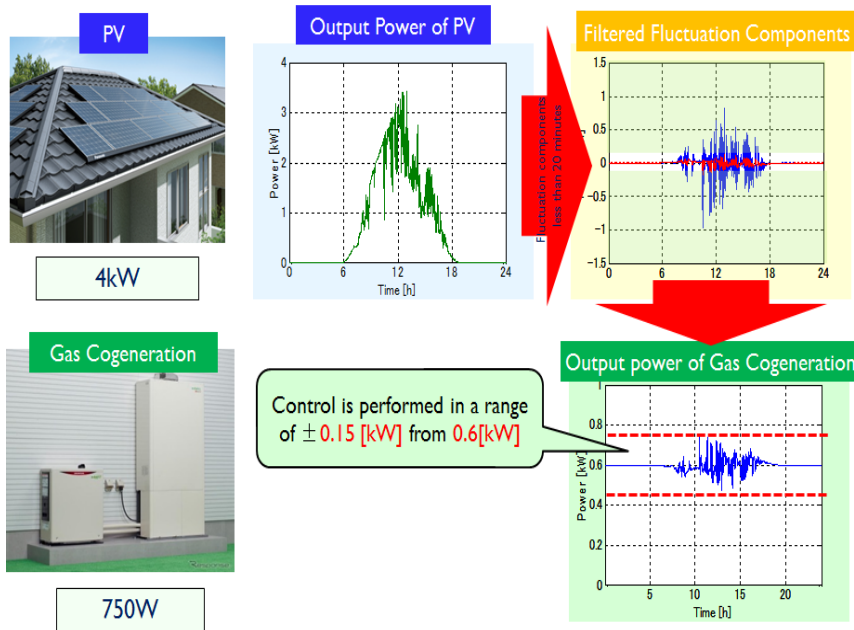

Fig. 5. The gas cogeneration system with a shrinkage factor in the load frequency power system model.

Fig. 6 and Fig. 7 show the result of the PV power output before and after the fluctuation, suppression control is applied. The proposed controlled applied in the gas cogeneration can be contribute to reduce PV fluctuations to some extent. Output of PV fluctuates very rapidly compared to Fig. 7.

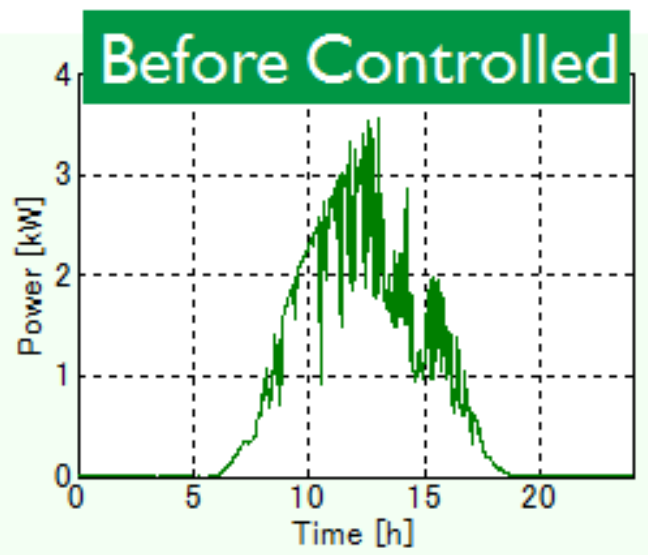

Fig. 6. The PV power output before controlled PV fluctuation.

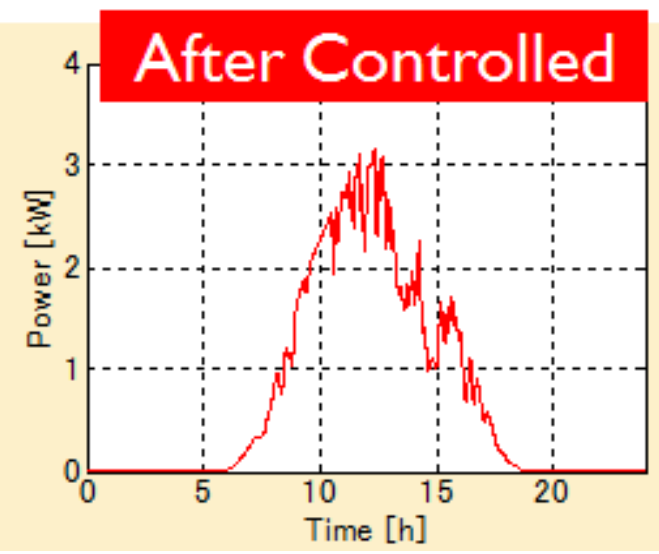

Fig. 7. The output after controlled PV fluctuation.
However, when the output of PV is too big compared to gas cogeneration output, it would be also difficult to control in the gas cogeneration and get a smooth output. When output of PV changes, the total efficiency of fluctuation suppression is shown in Fig. 8. The total amount of $15 \%$ depression effect can be gain by using a $4 \mathrm{~kW}$ of $\mathrm{PV}$ and $0.75 \mathrm{~kW}$ of gas cogeneration. The depression efficiency decreases gradually when output power of PV changes $0.5 \mathrm{~kW}$ to $4 \mathrm{~kW}$. Here, a direct proportional relationship can find between PV output and depression effect. For example, $90 \%$ of depression effect can be gain in $0.5 \mathrm{~kW}$ of PV where $4 \mathrm{~kW}$ of PV's depression effect reduces into $1 / 8$ compared to $0.5 \mathrm{~kW}$ PV. If $\mathrm{PV}$ output is more than $4 \mathrm{~kW}$, it is difficult to control the PV fluctuation by using $0.75 \mathrm{~kW}$ of the gas cogeneration.

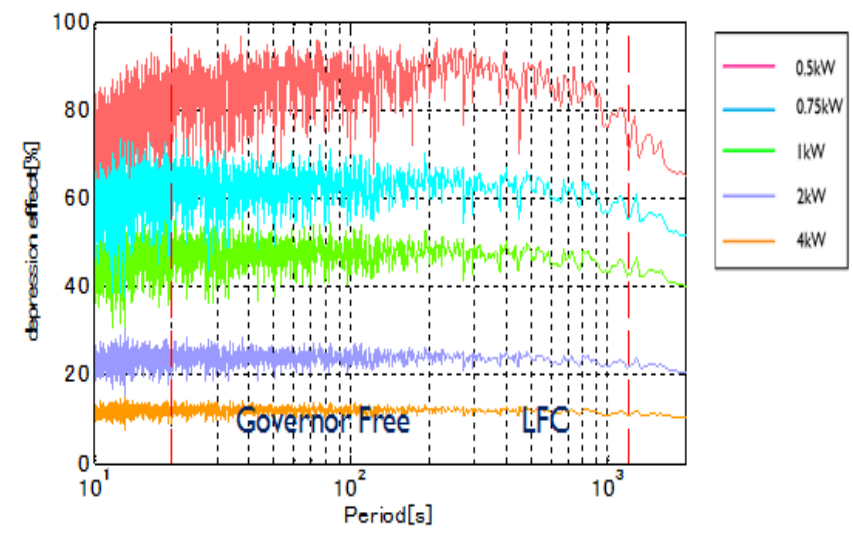

Fig. 8. Depression effect of the PV fluctuation.

\section{CONCLUSION}

The simulation results show that the domestic gas cogeneration systems are capable of suppressing the PV fluctuation and obtain a smooth power output, which is analyzed by the load frequency power system model. In this paper, the frequency fluctuation and power flowing to the power system are analyzed in detailed. But several challenges arise when the fluctuating PV output using the gas cogeneration such as introducing a large scale of PV to domestic where the output of gas cogeneration does not change and the PV fluctuation suppression in daytime when the domestic heat consumption is very low.

\section{REFERENCES}

[1] N. Belhaouas, M. S. A. Cheikh, A. Malek, and C. Larbes, "Matlab-simulink of photovoltaic system based on a two-diode model simulator with shaded solar cells," Revue des Energies Renouvelables, vol. 16 , no. 1 , pp. 65-73, 2013.

[2] R. J. Bessa, A. Trindade, C. S. P. Silva, and V. Miranda, "Probabilistic solar power forecasting in smart grids using distributed information," International Journal of Electircal Power and Energy Systems, vol. 72, pp. 16-23, 2015.

[3] Deployment of solar power in Japan: Economy, Trade and Industry Ministry of Japan. [Online]. Available: https://www.clarke-energy.com/chp-http://www.meti.go.jp/committee /materials2/downloadfiles/g90326a04j.pdf

[4] Cogeneration and CHP. [Online]. Available: https://www.clarke-energy.com/chpcogeneration/

[5] M. R. Benam, S. S. Madani, S. M. Alavi, and M. Ehsan, "Optimal configuration of the CHP system using stochastic programming," IEEE Transactions on Power Delivery, vol. 30, no. 3, pp. 1048-1056, June 2015.

[6] Domenstic gas cogeneration in ECO WILL system. [Online]. Availabe: http://www.honda.co.jpcogeneration/ 
[7] Passive high pass filter. [Online]. Available: http://www.electronics-tutorials.ws/filter/filter_3.html

[8] S. Haga, Y. Mitani, and M. Watanabe, "Analysis of mega-solar power fluctuation suppression by controllable distributed generation using power system model."

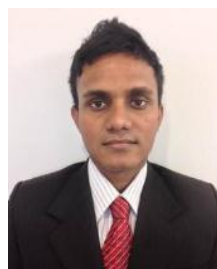

M. W. Saminda Roshan was born in Sri Lanka. He received his B.Sc. degree in electrical engineering from Kyushu Institute of Technology in 2015. Now he is working on his masters in electrical engineering degree at Kyushu Institute of Technology. His area of interest includes solar energies, cogeneration system, power systems modeling, stability and control.

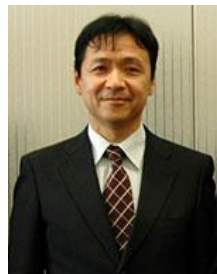

Yasunori Mitani received his B.Sc., M.Sc. and D.Eng. degrees in electrical engineering from Osaka University, Japan in 1981, 1983 and 1986, respectively. He was a visiting research associate at the University of California, Berkley, from 1994 to 1995. $\mathrm{He}$ is currently a professor at the Department of Electrical Engineering and Electronics, Kyushu Institute of Technology (KIT), Japan. At present, he is the head of the Environmental Management Center of KIT. His research interests are in the areas of analysis and control power systems. He is a member of the Institute of Electrical Engineers of Japan and IEE.

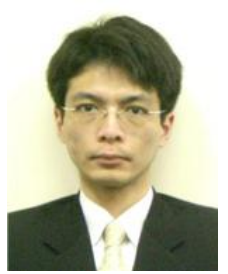

Masayuki Watanabe received the B.Sc., M.Sc., and D.Eng. degrees in electrical engineering from Osaka University, Japan, in 2001, 2002, and 2004, respectively. Currently, he is an associate professor at the Department of Electrical Engineering and Electronics, Kyushu Institute of Technology, Japan. His research interest is in the area of analysis of power systems.

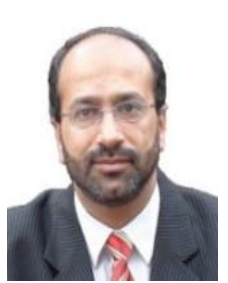

Yaser Soliman Qudaih graduated from the University of Engineering and Technology, Lahore, Pakistan in 1996 as an electrical engineer. $\mathrm{He}$ completed his M.Sc. and Ph.D. degrees from Kumamoto University, Japan in electrical engineering. $\mathrm{He}$ is currently a project assistant professor at the Department of Electrical Engineering and Electronics, Kyushu Institute of Technology(KIT)., Japan. His area of interest including power system is renewable energy and smart grid applications. He is a member of the Institute of Electrical Engineers of Japan and IEEE. 